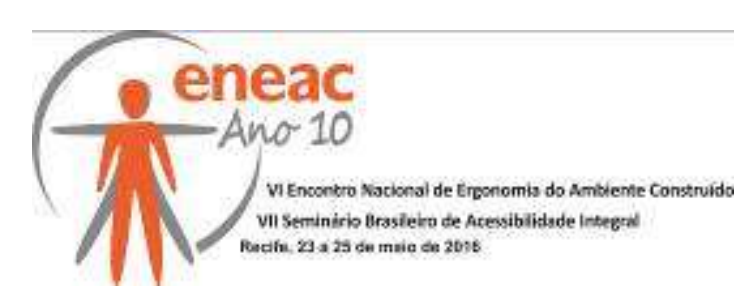

\title{
Cenário da produção científica brasileira sobre ergonomia do ambiente construído (2005 - 2015)
}

\author{
ARAÚJO, Maiana Cunha (1); \\ CAMPOS, Fabio (2); \\ VILLAROUCO, Vilma (3). \\ (1) UFPE, Mestrado em design \\ e-mail:maianacunha.ara@gmail.com \\ (2) UFPE, Doutorado em ciência da computação \\ e-mail:fc2005@gmail.com \\ (3) UFPE, Pós-doutorado em engenharia e gestão do conhecimento \\ e-mail:villarouco@hotmail.com
}

\begin{abstract}
RESUMO
A ergonomia do ambiente construído é uma disciplina científica cujo estudo e aplicação abrange diversas áreas do conhecimento, tratando diretamente com fatores do espaço edificado e suas interações com 0 indivíduo. Este tema tem se expandido gradativamente no meio científico internacional, e esta pesquisa tem por finalidade identificar a representatividade da produção científica brasileira sobre o tema em periódicos representativos e compreender suas origens.
\end{abstract}

Palavras chave: Ergonomia do ambiente construído, produção científica brasileira, periódicos, graduação, linhas de pesquisa.

\begin{abstract}
The ergonomics of the built environment is a scientific discipline witch study and application covers several areas of knowledge, dealing directly with factors of the built space and its interactions with the individual. This theme has expanded gradually in the international scientific community, and this research aims to identify the representative of the Brazilian scientific literature about this subject in representative journals and understand its origins.
\end{abstract}

Keywords: Ergonomics of the built environment, Brazilian scientific literature, journals , graduation, research lines.

\section{INTRODUÇÃO}

Segundo Soares (2006) a ergonomia deu seus primeiros passos no Brasil na década de 60 e desde então tem alcançado crescimento progressivo no país, desenvolvendo-se em associação com diversas áreas do conhecimento. Em agosto de 83 foi fundada a Associação Brasileira de Ergonomia (ABERGO) e com ela surgem os oito grupos técnicos que visam desenvolver os estudos em ergonomia em seus respectivos campos de atuação. Nesta ocasião oficializam-se os estudos em Ergonomia do Ambiente Construído (EAC), grupo técnico hoje gerido pela Profa. Dra. Vilma Villarouco, da Universidade Federal de 


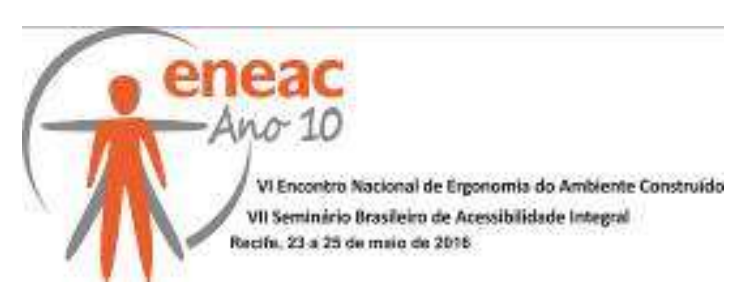

Pernambuco (UFPE) (http://abergo.org.br/). Como o próprio nome define, este ramo da ergonomia trata da sua relação com o espaço construído e como tudo isso se relaciona com o ser humano e suas atividades. Por esta relação com o espaço, este ramo da ergonomia tem grande relação com profissionais e pesquisadores da área de arquitetura e urbanismo.

O propósito central desta investigação é traçar o cenário atual da pesquisa em EAC no Brasil, traçando um perfil da produção científica nacional atual em periódicos de primeira linha da área do conhecimento em questão e compreender as bases desta produção investigando as matrizes curriculares de cursos de graduação em arquitetura e linhas de pesquisa relacionadas ao tema existentes no país.

O universo da pesquisa científica no mundo tem se tornado cada vez mais conectado e acessível. Com conteúdos disponibilizados digitalmente, pesquisadores do mundo todo podem trocar informações e divulgar seus trabalhos de forma mais abrangente ao acessar bases de dados, principalmente periódicos, que consistem em publicações eletrônicas ou impressas publicadas (como o próprio nome indica) periodicamente. O conteúdo publicado em periódicos chega mais rápido ao meio científico e é mais acessível, inclusive a longo prazo.

Nesta última plataforma, para identificar conteúdos com resultados e dados mais confiáveis, existem meios de classificação da produção intelectual. No Brasil uma métrica muito conhecida são as categorias Qualis, desenvolvidas pela Coordenação de Aperfeiçoamento de Pessoal de Nível Superior (Capes), fundação do Ministério da Educação (MEC). O Qualis é um conjunto de procedimentos utilizados pela Capes para avaliação da qualidade da produção científica encontrada nos periódicos, classificando os de maior qualidade como A1 e sucessivamente decrescendo para A2, B1, B2, etc (http://www.periodicos.capes.gov.br/).

Existem, porém, outras métricas utilizadas pelos pesquisadores como referência. $O$ fator de impacto (FI) oferece uma perspectiva para avaliação e comparação de periódicos por meio da acumulação e tabulação de contagens de citações e artigos e é usado para comparar diferentes periódicos de uma determinada área do conhecimento. Os FI são calculados anualmente para os periódicos indexados ao Institute of Scientific Information (ISI) e depois publicados no Journal of Citations Report (JCR) (ANDRADE; PINTO, 1999). Apresenta dados quantitativos que apoiam uma revisão sistemática e objetiva das revistas cobrindo as seguintes áreas do conhecimento: Ciências Agrárias, Ciências Biológicas, Ciências Sociais, Ciências Sociais Aplicadas, Ciências Exatas, Ciências da Terra e Engenharias. (REUTERS, 2012)

Para esta pesquisa foram considerados periódicos classificados como pertencentes à área do conhecimento "Ciências Sociais Aplicadas", subgrupos "Arquitetura e Urbanismo" e "Engenharias III", onde encontram-se as publicações referentes à EAC. Foram selecionados para a pesquisa periódicos da categoria Qualis A1, A2 e B1 que possuem FI representativo na área em questão.

\section{CONCEITOS BÁSICOS}

A definição oficial de ergonomia, dada pela International Ergonomics Association (IEA) e divulgada pela associação Brasileira de Ergonomia (ABERGO) diz que "é disciplina relacionada ao entendimento das interações entre os seres humanos e outros elementos ou sistemas". Segundo lida (2005), ergonomia é o estudo da adaptação do trabalho ao homem, sendo "trabalho" um termo que refere-se a qualquer atividade produtiva exercida, não implicando, necessariamente, no envolvimento de uma máquina. "A ergonomia tem urna visão ampla, abrangendo atividades de planejamento e projeto, que ocorrem antes do trabalho ser realizado, e aqueles de controle e avaliação, que ocorrem durante e após esse 


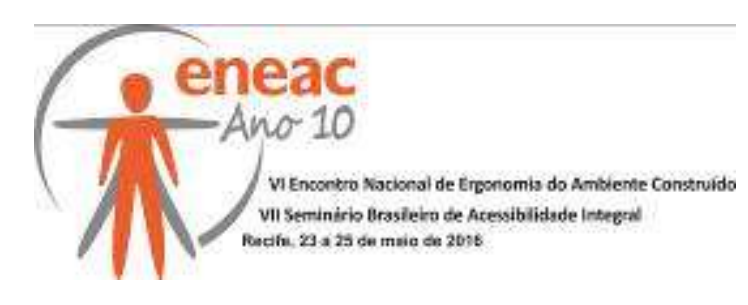

trabalho". Na atividade ergonômica, tudo o que envolve a realização de uma tarefa precisa ser visto e projetado para o usuário, inclusive o espaço arquitetônico.

Quando se trata de EAC, entende-se que seu objeto de estudo é a interação entre o homem e o espaço, e segundo Guidalli (2012) o espaço construído é a concretização de um projeto de edificação, "quando este se torna um objeto edificado, presente no mundo, com funções práticas e estruturado em técnicas construtivas". De acordo com Zevi (1996), "ambiente construído será aquele que remete ao espaço arquitetônico, o ambiente da convivência humana".

Mont'Alvão (2011) destaca que ao tratar de ergonomia do ambiente construído fica evidente a correlação entre conceitos bastante distintos: o espaço construído propriamente dito e a ergonomia. Porém ressalta que, unindo a ideia de ambiente arquitetônico com o ambiente do desenvolvimento das tarefas, de acordo com as capacidades, habilidades e limitações humanas, parece clara a necessidade dos conhecimentos da ergonomia nos projetos de arquitetura e design que contemplam o ambiente construído, porque embora apresentando preocupações naturais de outras áreas do conhecimento, a ergonomia do ambiente extrapola as questões puramente arquitetônicas, focando o seu posicionamento na adaptabilidade e conformidade do espaço às tarefas e atividades que neles irão se desenvolver.

Villarouco (2002) refere que ao considerar os diversos aspectos envolvidos no ambiente construído, há a necessidade de uma abordagem sistêmica. Para a autora, uma completa avaliação ergonômica do ambiente abrange variáveis como conforto ambiental (lumínico, térmico, acústico), percepção ambiental (aspectos cognitivos), antropometria (acessibilidade, dimensionamento) e adequação de materiais (revestimentos, acabamentos). Essa avaliação do ambiente construído leva à compreensão de que o ato de projetar deve ter como principal norteador o homem, com toda a sua bagagem vivencial, com sua sensibilidade, seu funcionamento biológico, sua percepção, seu comportamento, pois o produto do fazer projetual, invariavelmente, destina-se a abrigar o ser humano executando tarefas.

Pela própria natureza da EAC, seu estudo está muito relacionado com a prática profissional e desenvolvimento científico de profissionais da área de arquitetura e urbanismo, apesar de não se limitar a eles, mas consequentemente as publicações sobre o tema estão presentes em periódicos da área, assim como os do subgrupo "Engenharia III", onde, de acordo com a classificação da CAPES, estão locados periódicos relativos à ergonomia.

\section{TÉCNICAS ADOTADAS}

Para o desenvolvimento desta pesquisa foram adotados as seguintes etapas:Os trabalhos serão avaliados segundo os seguintes critérios:

- Pesquisa na página do Qualis CAPES (https://qualis.capes.gov.br) pela lista de periódicos classificados em 2014 (última classificação disponível até a presente data) nas categorias $A 1, A 2$ e $B 1$;

- Com a lista dos periódicos, foram inclusos na pesquisa somente periódicos que dispunham do fator de impacto JCR;

- A partir dos periódicos selecionados, se mantiveram inclusos na pesquisa aqueles que apresentaram resultados para a busca pelas seguintes palavras-chave e combinações: ergonomics, ergonomics AND "built environment", ergonomics AND architecture. 


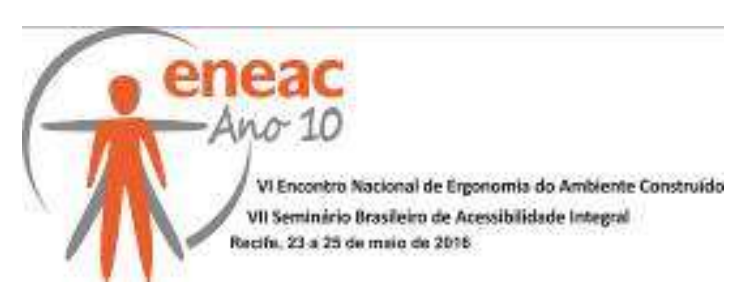

A partir dos resultados obtidos, foi feita uma nova triagem nos artigos encontrados, com base na análise dos títulos, resumos e conclusões, com a finalidade de excluir artigos que não tratavam da relação ergonomia e ambiente construído. Também foi adotado como critério de inclusão a data de publicação do artigo, selecionando somente os que foram publicados nos últimos 10 anos (2005 a 2015).

O resultado desta triagem possibilitou a seleção das publicações de pesquisadores vinculados a instituições de ensino brasileiras, identificando as origens destas publicações e os temas mais tratados, levando à necessidade de compreensão do cenário da pesquisa sobre EAC no país. Com isto observou-se a predominância de pesquisadores relacionados a instituições públicas de ensino, assim como participantes de linhas de pesquisa nestas instituições.

A partir disto, foram adotadas duas frentes de abordagem:

- Busca por linhas de pesquisa relacionadas ao tema, vinculadas ao CNPq (Conselho Nacional de Desenvolvimento Científico e Tecnológico) através de busca na página do conselho (lattes.cnpq.br/web/dgp) pelas seguintes palavras-chave: ergonomia AND ambiente;

- Investigação das matrizes curriculares dos cursos de graduação em arquitetura e urbanismo oferecidos por instituições públicas de ensino, com a finalidade de identificar a oferta ou não da disciplina de ergonomia.

Com os dados obtidos nestas duas etapas foi possível compreender a relação entre a base da formação do pesquisador da área de EAC com a produção científica publicada em periódicos considerados representativos no meio acadêmico.

\section{RESULTADOS}

\subsection{Publicações em periódicos}

De uma amostragem inicial de 86 periódicos, foram selecionados 17 para consulta de artigos, sendo adotados como critérios de exclusão os fatores descritos anteriormente, como ter um fator de impacto representativo e pertencer a uma boa categoria no sistema Qualis/CAPES. Os 17 periódicos são:

Tabela 1 - Lista de periódicos pesquisados com respectivas métricas.

\begin{tabular}{|l|c|c|}
\hline \multicolumn{1}{|c|}{ PERIÓRICO } & $\begin{array}{c}\text { FATOR DE } \\
\text { IMPACTO }\end{array}$ & QUALIS/CAPES \\
\hline Journal of Cleaner Production & 3,844 & $\mathrm{~A} 1$ \\
\hline Building and Environment & 3,341 & $\mathrm{~A} 1$ \\
\hline Energy and Buildings & 2,884 & $\mathrm{~A} 1$ \\
\hline Energy Policy & 2,575 & $\mathrm{~A} 1$ \\
\hline International Journal of Fatigue & 2,275 & $\mathrm{~A} 1$ \\
\hline Expert Systems with Applications & 2,240 & $\mathrm{~A} 1$ \\
\hline Applied Ergonomics & 2,023 & $\mathrm{~A} 2$ \\
\hline
\end{tabular}




\begin{tabular}{|c|c|c|}
\hline Habitat International & 1,746 & A1 \\
\hline Natural Hazards & 1,719 & A1 \\
\hline Human Factors & 1,694 & B1 \\
\hline Indoor + built environment & 1,225 & B1 \\
\hline International Journal of Industrial Ergonomics & 1,070 & $\mathrm{~A} 1$ \\
\hline International Journal of Industrial Ergonomics & 1,070 & A1 \\
\hline Ciência e Saúde Coletiva & 1,030 & $\mathrm{~A} 2$ \\
\hline Futures & 1,012 & $\mathrm{~A} 2$ \\
\hline IEEE Computer Graphics and Applications & 0,911 & A1 \\
\hline Journal of Housing and the Built Environment & 0,657 & B1 \\
\hline
\end{tabular}

Todos os periódicos listados foram consultados, sendo feitas buscas com as palavras-chave já mencionadas. Dos 533 artigos gerados na busca, apenas 300 foram selecionados para entrar como material de pesquisa (após passar pela triagem de título, resumo e conclusão) por tratarem da relação entre ergonomia e ambiente construído.

Durante a leitura deste material foi possível relacionar as publicações com os países de origem, gerando um retrato da atuação dos pesquisadores de EAC brasileiros no cenário mundial.

\section{Gráfico 1 - Percentual de publicações por país.}

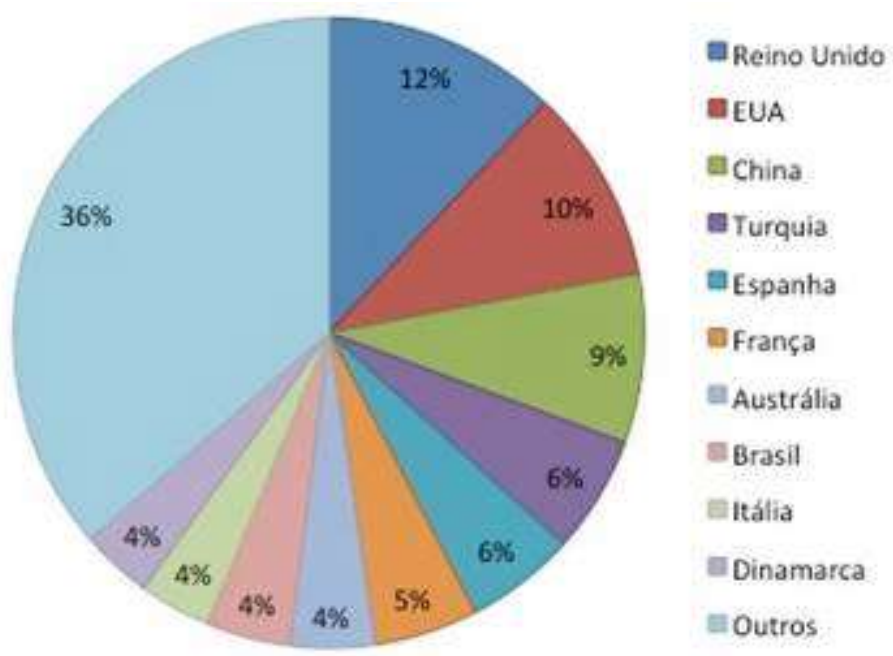

Os principais temas abordados pelos países em geral tratavam da a ergonomia no ambiente construído e a sua interferência na saúde do trabalhador, relacionando com frequência o tema a fatores de conforto ambiental (como acústica, temperatura, luminosidade) e percepção (cognição e percepção ambiental). Os ambientes abordados por estes artigos foram: ambientes industriais, hospitalares, salas de aula, ambientes de aprendizado (laboratórios de informática, bibliotecas, etc), ambientes corporativos, canteiros de obras e instituições de cuidados com idosos, evidenciando a profunda relação homem-atividadeambiente.

Nas publicações brasileiras, entretanto, observou-se a predominância dos seguintes temas (sem entretanto, haver predominância de um sobre outros):

- Ergonomia e sustentabilidade - tratando da relação colaborativa entre edifícios sustentáveis e princípios da ergonomia;

- Ergonomia da informação - relacionando a ergonomia dos rótulos de medicamentos manipulados em enfermarias de hospitais. Fatores ambientais entraram como agravantes da fadiga e baixa acuidade visual; 


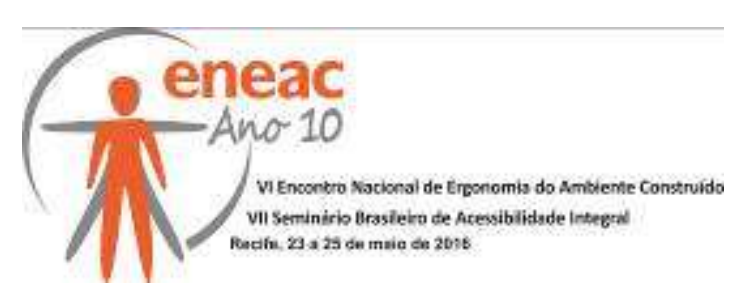

- Ergonomia em sistemas de produção - o ambiente entra como fator de avaliação do posto de trabalho em indústrias;

- História da ergonomia - com ênfase na américa latina e especificamente no Brasil;

- Ergonomia em salas de aula - relacionando ambiente, ergonomia e aprendizagem. Tema que foi observado como sendo uma tendência de pesquisa em publicações internacionais;

- Saúde do trabalhador - fazendo a relação entre altos índices de absenteísmo com a má qualidade dos ambientes de trabalho.

As publicações brasileiras originaram-se de pesquisadores vinculados a instituições públicas presentes nos seguintes estados: São Paulo, Minas Gerais, Rio Grande do Sul, Rio de Janeiro, Pernambuco, Paraná, Distrito Federal e Bahia.

\subsection{Ergonomia nos cursos de graduação}

Com o dados apurados na revisão dos artigos, identificou-se a necessidade de estudar a base da formação dos pesquisadores da área. Para tanto, foram estudadas as matrizes curriculares dos cursos de graduação em arquitetura e urbanismo ofertados por instituições públicas. A intenção é verificar se o contato com o tema EAC acontece ainda na graduação, onde a base do conhecimento do profissional da área de arquitetura está sendo formado.

De acordo com pesquisa realizada na página eletrônica do ministério da educação (http://emec.mec.gov.br/) até o ano de 2015 haviam 466 cursos de graduação em arquitetura e urbanismo sendo ofertados em 27 unidades da federação. Destes, 47 são oferecidos em instituições públicas, representando pouco mais de $10 \%$ dos cursos ofertados no país. Destes 47, 12 não fornecem em suas páginas a matriz curricular do curso, o que impossibilitou uma análise mais exata da questão, porém foi possível averiguar que 29 instituições não oferecem a disciplina, 03 oferecem como disciplina eletiva (ou optativa) e 03 possuem a disciplina de ergonomia, tratando especificamente da ergonomia do ambiente construído, em seu quadro de disciplinas obrigatórias do curso.

As três instituições que oferecem a disciplina de ergonomia como eletiva (ou optativa) são a UFAL (Alagoas), UNIFAP (Amapá) e UNB (Distrito Federal), e as três instituições que incluíram ergonomia como disciplina obrigatória em seus cursos foram a UNILA (Paraná), UFPE (Pernambuco) e USP (São Paulo), sendo que esta última oferece a disciplina em dois módulos.

Pela falta de informação acerca de 12 dos cursos, não é possível ser taxativo quando à falta de contato dos alunos de arquitetura com o tema EAC, porém com o que foi apurado, acredita-se que poderá haver um potencial desenvolvimento de pesquisas na área se forem aumentadas as possibilidades de contato com a ergonomia ainda na graduação.

\subsection{Linhas de pesquisa registradas no CNPq}

A etapa seguinte da pesquisa visou identificar linhas de pesquisa que tratam do tema EAC. Para tanto, foi feita uma consulta à página do CNPq (lattes.cnpq.br/web/dgp) seguindo os seguintes parâmetros: consulta por linha de pesquisa, usando como termos de busca as palavras "ergonomia" e "ambiente", podendo ser identificados como nome do grupo, nome da linha de pesquisa, palavra-chave da linha de pesquisa e/ou objetivo da linha de pesquisa, e considerando somente os grupos certificados pelo CNPq. 


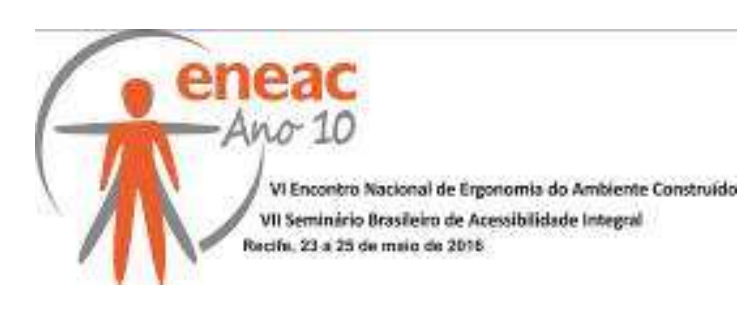

Da pesquisa com estes parâmetros resultaram 24 linhas de pesquisa que distribuem-se do nordeste ao sul do país. Na imagem abaixo, os estados destacados em azul são os que possuem linhas de pesquisa que tratam em algum aspecto do tema ergonomia do ambiente construído.

Dos estados em destaque, é importante informar que Bahia, Maranhão, Paraíba, Paraná, Pernambuco, Rio Grande do Sul e Santa Catarina possuem linhas de pesquisa que tratam especificamente do tema ergonomia do ambiente construído. Identificar as linhas de pesquisa foi fundamental para o cruzamento de algumas informações que resultaram em dados conclusivos para esta investigação.

Imagem 1 - Mapa com destaque para os estados com linhas de pesquisa em EAC.

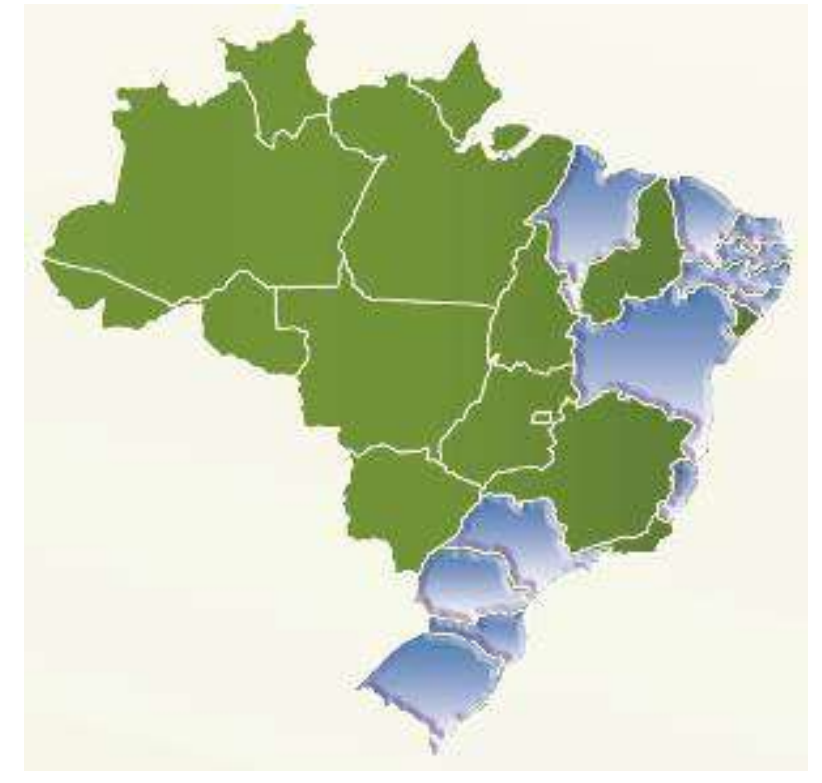

\subsection{Questões em aberto}

Com relação à investigação acerca dos artigos publicados em periódicos, acredita-se que para uma identificação mais específica sobre a produção científica nacional da área, sobre quais temas tem sido explorados pelos pesquisadores das instituições brasileiras, será necessária a utilização de parâmetros diferentes dos adotados neste relato, pois a representatividade dos artigos brasileiros (apenas 4\%) em periódicos representativos é baixa para a definição de um cenário nacional real.

Sobre a questão da oferta da disciplina de ergonomia, acredita-se que a falta de informação acerca da matriz curricular de 12 dos cursos deixou o resultado deste aspecto da pesquisa inconclusivo, e para considerar o real cenário da ergonomia na esfera da graduação considera-se necessária a consulta de matrizes curriculares também dos cursos ofertados por instituições privadas, que representam mais de $89 \%$ dos cursos de arquitetura do país. Para os fins desta pesquisa foram adotados somente aqueles ofertados por instituições públicas pela relação com as linhas de pesquisa e com as instituições relacionadas nas publicações dos periódicos analisados.

\section{CONCLUSÃO}

Ao relacionar as origens das publicações em periódicos representativos, com os cursos de graduação que oferecem disciplinas de ergonomia (optativas ou obrigatórias) e com a 


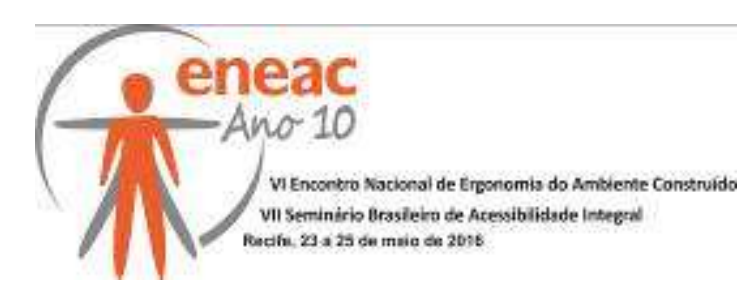

localização das linhas de pesquisa em EAC identificou-se que, dos sete estados relatados como origens das publicações, quatro possuem linhas de pesquisa na área, e coincidentemente os quatro oferecem a disciplina para os alunos da graduação (sendo que desses quatro, três são os que oferecem ergonomia como disciplina obrigatória).

Observa-se, com isso, que a presença do conteúdo acerca da ergonomia do ambiente construído na graduação, somada à possibilidade de expansão do conhecimento através das linhas de pesquisa levou a produção científica brasileira a figurar entre os periódicos de influência no meio científico. Acredita-se, com isso, que o aumento da representatividade internacional da produção brasileira pode estar relacionada com o aumento do investimento nas bases do desenvolvimento acadêmico.

No caso específico da EAC, sua atuação não se restringe aos profissionais de arquitetura, mas devido à profunda relação que tem com o espaço edificado, profissionais e pesquisadores desta área podem colaborar de forma muito positiva para o desenvolvimento deste campo. A inserção da ergonomia como componente da formação base do arquiteto, além de formar profissionais mais capacitados, forma futuros pesquisadores com bases mais sólidas, mais preparados para trabalhar com o meio científico.

É importante destacar que as possíveis expansões desta pesquisa (relacionadas no item 4.4) levarão a resultados mais conclusivos e consistentes sobre o tema aqui tratado, porém o conteúdo abordado já permitiu traçar possíveis caminhos para uma produção acadêmica e científica brasileira mais representativa internacionalmente, além de colaborar para o desenvolvimento de um tema cujo principal motor impulsionador a melhoria da qualidade de vida do ser humano.

\section{REFERÊNCIAS BIBLIOGRÁFICAS}

ANDRADE, Jailson B.; PINTO, Angelo C. Fator de impacto de revistas científicas: qual o significado deste parâmetro? In: Química Nova, Volume 22, 1999. Páginas 448-453.

GUIDALLI, C. R. R. Diretrizes para o projeto de salas de aula em universidades visando o bem estar do usuário. 2012. 237f. Dissertação (Mestrado em Arquitetura e Urbanismo). Universidade Federal de Santa Catarina. Florianópolis, 2012.

IIDA, I. Ergonomia: projeto e produção. 2 ed. São Paulo: Edgard Blücher, 2005.

MONT'ALVÃO, C. A ergonomia do ambiente construído no Brasil. In: MONT'ALVÃO, C.; VILLAROUCO, V. Um novo olhar para o projeto: a ergonomia no ambiente construído. Teresópolis: 2AB, 2011. p. 13-24.

REUTERS, Thomson. Impact factor. Journal Citation Reports. 2012b. Disponível em: Acesso em: $<$ http://admin-apps.webofknowledge.com/JCR/help/h_impfact.htm\#impact_factor> Acesso em 06/12/2015.

SOARES, Marcelo M. Ergonomics in latin américa: background, trends and challenges. In Applied Ergonomics, Volume 37, 2006. Páginas 555-561.

VILLAROUCO, V. Avaliação ergonômica do projeto arquitetônico. Anais do ABERGO 2002 - VI Congresso latino-americano de Ergonomia e XII Congresso Brasileiro de Ergonomia. Recife, 2002.

ZEVI, B. Saber ver a arquitetura. Tradução de Maria Isabel Gaspar e Gaëtan Martins de Oliveira. 5aed. São Paulo: Martins Fontes, 1996. 


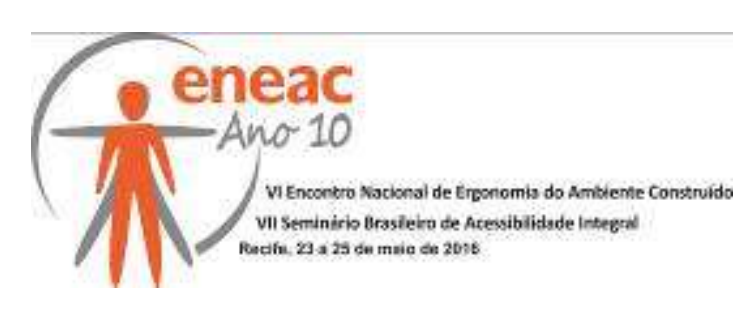

\section{REFERÊNCIAS ELETRÔNICAS}

ABERGO - Associação Brasileira de Ergonomia. Disponível em: <http://abergo.org.br/>. Acesso em 16/12/2015.

Applied Ergonomics. Disponível em < http://www.journals.elsevier.com/applied-ergonomics> . Acesso em 11/12/2015.

Building and Environment. Disponível em <http://www.journals.elsevier.com/building-andenvironment/> . Acesso em 16/12/2015.

CAPES - Coordenação de Aperfeiçoamento de Pessoal de Nível Superior. Disponível em < http://www.periodicos.capes.gov.br/>. Acesso em 09/12/2015.

Energy and Buildings. Disponível em <http://www.journals.elsevier.com/energy-and-buildings/>. Acesso em 16/12/2015.

Energy Policy. Disponível em <http://www.journals.elsevier.com/energy-policy/Fator de impacto: 2.575>. Acesso em 16/12/2015.

Expert Systems with Applications. Disponível em <http://www.journals.elsevier.com/expertsystems-with-applications/>. Acesso em 16/12/2015.

Habitat International. Disponível em <http://www.journals.elsevier.com/habitat-international/>. Acesso em 18/12/2015.

Human Factors. Disponível em < http://hfs.sagepub.com.ez16.periodicos.capes.gov.br/content/by/year

>. Acesso em 11/12/2015.

IEEE Computer Graphics and Applications. Disponível em $<$ http://ieeexplore.iee. $.0 \mathrm{rg} / \mathrm{xpl} /$ Recentlssue.jsp?punumber=38>. Acesso em 18/12/2015.

Indoor + built environment. Disponível em <http://ibe.sagepub.com.ez16.periodicos.capes.gov.br/content/by/year>. Acesso em 11/12/2015.

International Journal of Fatigue. Disponível em <http://www.journals.elsevier.com/internationaljournal-of-fatigue/>. Acesso em 16/12/2015.

International Journal of Industrial Ergonomics. Disponível em $<$ http://www.journals.elsevier.com/international-journal-of-industrial-ergonomics>. Acesso em $11 / 12 / 2015$.

Journal of Cleaner Production. Discponível em <http://www.journals.elsevier.com/journal-of-cleanerproduction/>. Acesso em 16/12/2015.

Journal of Housing and the Built Environment. Disponível em $<$ http://link.periodicos.capes.gov.br.ez16.periodicos.capes.gov.br/sfxlcl41?url_ver=Z39.882004\&url_ctx_fmt=fi/fmt:kev:mtx:ctx\&ctx_enc=info:ofi/enc:UTF-8\&ctx_ver=Z39.882004\&rfr_id=info:sid/sfxit.com:azlist\&sfx.ignore_date_threshold=1\&rft.object_id=963017857338\&svc.f ulltext=yes>. Acesso em 11/12/2015.

Natural Hazards. Disponível em <http://link.springer.com/journal/11069>. Acesso em 18/12/2015. 